\title{
Inatividade física no deslocamento para a escola e fatores associados em adolescentes de uma cidade do Sul do Brasil
}

http://dx.doi.org/10.11606/1807-5509202000010123

\author{
André de Araújo PINTO* \\ Diego Augusto Santos SILVA** \\ Kelly Samara SILVA** \\ Érico Pereira Gomes FELDEN* \\ Andreia PELEGRINI*
}

*Universidade do Estado de Santa Catarina, Florianópolis, SC, Brasil.

**Departamento de Educação Física, Universidade Federal de Santa Caratina, Florianópolis, SC, Brasil.

\section{Resumo}

O objetivo deste estudo foi estimar a prevalência de inatividade física no deslocamento para a escola e identificar os fatores associados em adolescentes de uma cidade do Sul do Brasil. Estudo epidemiológico, conduzido em 1.029 adolescentes de 15 a 19 anos, de escolas públicas estaduais da cidade de São José-SC. Um questionário foi aplicado com intuito de obter as informações referentes à variável dependente, deslocamento passivo para a escola (carro/moto, ônibus) e as variáveis independentes, sendo as características sociodemográficas (sexo, faixa etária e renda familiar mensal), atividade física e tempo gasto no deslocamento. A massa corporal e a estatura foram mensuradas para a determinação do status do peso por meio do IMC. Empregou-se a regressão logística binária, estimando-se a razão de Odss (R0) para identificar a associação entre a variável dependente e as demais variáveis. A prevalência de deslocamento passivo entre os adolescentes foi de 52\%. Os rapazes mais velhos (17-19 anos), os adolescentes de famílias com maior renda familiar mensal (> 5 salários), àqueles que não atenderam as recomendações de atividade física e os que gastaram maior tempo no deslocamento ( $\geq 20$ minutos para os rapazes e $\geq 10$ minutos para as moças) apresentaram mais chances de se deslocarem passivamente para a escola. Mais da metade dos adolescentes se deslocam de maneira passiva no trajeto casa-escola. Os grupos mais expostos ao deslocamento passivo são os rapazes mais velhos, os adolescentes com maior renda familiar, que não atendem às recomendações mundiais de atividade física e despendem maior tempo no deslocamento.

Palavras-chave: Atividade Física; Caminhada; Ciclismo; Saúde do Adolescente.

\section{Introdução}

A inatividade física (IAF), associada às principais doenças crônicas não transmissíveis como diabetes tipo 2 e doença cardíaca coronária tem sido responsável por, aproximadamente, cinco milhóes de mortes anualmente ${ }^{1}$. No mundo, estima-se que $80,3 \%$ dos adolescentes de 13 a 15 anos praticam menos de 60 minutos diários de atividade física (AF) moderada/vigorosa ${ }^{2}$. Com base nisso, diversas entidades de saúde pública de diferentes partes do mundo têm se reunido para desenvolver estratégias visando à redução da prevalência de $\mathrm{IAF}^{3}$. Dentre elas, o deslocamento ativo, considerado um dos domínios da AF, tem sido sugerido como um hábito que pode contribuir para o atendimento das recomendações de AF diária ${ }^{4}$.

Revisões sistemáticas recentes demostraram, maiores riscos na saúde cardiovascular, excesso de peso corporal ${ }^{5}$, e diabetes ${ }^{6}$ em escolares que se deslocavam passivamente para a escola. Em revisão sistemática conduzida por LAROUCHE, et al. ${ }^{5}$ com crianças e adolescentes, foi observado na maior parte dos estudos menores níveis de aptidão cardiorrespiratória e de AF, composição corporal pouco saudável, entre aqueles que se deslocavam de maneira passiva para a escola. Isso de fato merece maiores esclarecimentos, principalmente nos 
adolescentes, tendo em vista que o poder de decisão no processo de escolha no modo de transporte utilizado no deslocamento de casa até à escola entre eles é maior, do que entre as crianças ${ }^{4}$.

Neste contexto observa-se um interesse cada vez maior de estudiosos da área da saúde em investigar o comportamento dos adolescentes em relação ao tipo de deslocamento utilizado para ir à escola ${ }^{4-13}$. A este respeito, muitos países têm verificado elevadas prevalências de deslocamento passivo para a escola destacando-se os Estados Unidos $(95,0 \%)^{9}$, Portugal $(78,6 \%)^{12}$ e Alemanha $(60,0 \%)^{10}$. No Brasil, prevalências do deslocamento passivo variaram de $(35,3 \%)$ adolescentes do Rio Grande do $\mathrm{Sul}^{11}$ a $(43,3 \%)$ de Santa Catarina ${ }^{6}$.

Além das elevadas prevalências encontradas, a literatura reporta que muitos estudos têm tentado identificar os adolescentes mais expostos à ocorrência deste evento, destacando-se aqueles com idade mais avançada ${ }^{4,10}$, provenientes de famílias com maiores condições financeiras ${ }^{6,7,10,11}$ e os adolescentes que moram mais longe da escola $^{6,14}$. Essas evidências reforçam a necessidade de novos estudos sobre a temática, principalmente no Brasil, por se tratar de um país em constantes transformações socioeconômicas.

Além disso, e dos relatos na literatura sobre a importância do modo deslocamento ativo nos diferentes aspectos de saúde dos adolescentes, é aceitável que aqueles que estão mais expostos ao deslocamento passivo para a escola necessitam de maior atenção, principalmente por não disporem dos possíveis benefícios para a saúde ao alcance do deslocamento ativo, e estarem mais vulneráveis aos desfechos desfavoráveis à saúde. Portanto, investigar o tipo de deslocamento utilizado para ir à escola poderá contribuir para o melhor entendimento sobre o engajamento dos adolescentes em atividades físicas, e ainda orientar o desenvolvimento de estratégias que visem promover o deslocamento ativo contribuindo para um estilo de vida ativo. Nesse sentido, o objetivo do presente estudo foi estimar a prevalência de IAF no deslocamento para a escola e identificar os fatores associados em adolescentes de uma cidade do Sul do Brasil.

\section{Método}

Este estudo transversal, de caráter epidemiológico, é baseado em uma análise secundária de dados vinculados ao projeto "Guia Brasileiro de Avaliação da Aptidão Física Relacionada à Saúde - Etapa 1”, no qual é fruto de uma parceria entre a Universidade Federal de Santa Catarina, Universidade do Estado de Santa Catarina, Universidade Federal de Sergipe e University of Ottawa/Canadá. Esta pesquisa foi aprovada pelo Comitê de Ética em Pesquisa com Seres Humanos da Universidade Federal de Santa Catarina (Parecer no 746.536/2014).

São José, um dos 295 municípios de Santa Catarina, está situado na região da Grande Florianópolis. Localizado na região litorânea do estado, o município é o único que faz divisa terrestre com a capital do estado, Florianópolis, possuindo 28 bairros que compõe seus três distritos (Campinas, Barreiros e São José) com peculiaridades distintas, no que diz respeito aos aspectos culturais, sociais e econômicos. Seu Índice de Desenvolvimento Humano Municipal (IDHM) de 0,809, o coloca como quarto município do estado com melhor IDHM $^{15}$. São José apresenta um percentual de $70,94 \%$ de jovens (15-17 anos) com ensino fundamental completo, possui renda per capita de R\$ $1.157,43$ e possui um índice Gini $(0,44)$ que retrata baixa desigualdade de renda entre os habitantes ${ }^{15}$.

A população-alvo do macro estudo limitou-se aos estudantes das escolas públicas estaduais do ensino médio do município de São José, com idade de 14 a 19 anos. Segundo a Secretaria de Estado da Educação de Santa Catarina, 5.182 estudantes estavam regularmente matriculados no ensino médio, em 11 escolas elegíveis para o presente estudo, distribuídos em 170 turmas no ano de 2014. Os procedimentos sugeridos por LUIZ e MAGNANINI ${ }^{16}$ foram utilizados para definir o tamanho amostral. Para tal, adotou-se um erro tolerável de cinco pontos percentuais, nível de significância de 1,96 (IC95\%), prevalência de $50 \%$ (desfecho desconhecido) e efeito de delineamento de 1,5. Após esses parâmetros, optou-se por acrescentar $20 \%$ para minimizar eventuais perdas relacionadas às possíveis recusas $\mathrm{e}$ mais $20 \%$ para o controle de possíveis variáveis de confusão, normalmente acrescentados em estudos de associação ${ }^{17}$. Considerando-se todos esses critérios, seria necessário alcançar uma amostra de 751 
adolescentes. Devido ao processo de conglomerado de turmas em que foram coletados todos os alunos das turmas sorteadas, a amostra foi totalizada em 1.148 estudantes. Para o presente estudo considerou adolescentes apenas os estudantes com idades de 15 a $19 \operatorname{anos}^{18}$.

Para a determinação da amostra, recorreu-se a um processo de amostragem determinado em dois estágios: primeiro estratificado por escolas públicas estaduais de ensino médio e posteriormente por conglomerado de turmas, considerando turnos, séries e turmas. No primeiro estágio adotou-se como critério de estratificação a densidade da escola (pequenas, com menos de 200 alunos; médias, com 200 a 499 alunos; e grandes, com 500 estudantes ou mais), deste modo, o sorteio foi realizado, proporcionalmente, de acordo com o porte. Para o segundo estágio, foi considerado o turno de estudo, e a série de ensino. Todos os estudantes matriculados nas turmas sorteadas foram convidados a participar do estudo independentemente da idade deles.

Os estudantes das turmas sorteadas e que estavam presentes no dia da coleta, foram informados sobre os objetivos da pesquisa e posteriormente foram convidados a participarem da mesma. Aqueles estudantes que aceitaram participar voluntariamente, que assinaram o Termo de Assentimento e apresentaram o Termo de Consentimento Livre e Esclarecido assinados pelos pais/responsáveis (para os adolescentes com idade $<18$ anos) ou por eles mesmos (idade $\geq 18$ anos) foram incluídos no presente estudo.

\section{Variáveis do Estudo}

A variável dependente, deslocamento para a escola foi avaliada por meio da seguinte pergunta: "Como normalmente você se desloca para ir à escola (colégio)?”. As possíveis respostas (“a pé”, "bicicleta", "por carro ou moto", "ônibus" ou "outros") foram categorizadas em deslocamento ativo (a pé ou bicicleta) e deslocamento passivo (carro, moto ou ônibus). Além disso, buscouse identificar o tempo gasto no percurso entre a residência e a escola por meio da pergunta "Quanto tempo você gasta nesse deslocamento?", com a resposta em faixas de minutos por dia: $<10$ minutos/dia, 10 a 19 minutos/dia, 20 a 29 minutos/ dia, 30 a 39 minutos/dia, 40 a 49 minutos/dia, 50 a 59 minutos/dia, 60 minutos ou mais (SiLVA et al., 2013). Para fins de análise, optou-se por agrupar as categorias em a) menos de 10 minutos, b) entre 10 e 19 minutos e c) 20 minutos ou mais ${ }^{19}$.

Foram coletadas informaçōes referentes ao sexo (masculino e feminino), idade (15-16 anos e 17-19 anos), turno (diurno e noturno) e série de estudo (primeiro, segundo e terceiro ano). A renda familiar foi estimada com base no salário mínimo vigente em 2014 ( $\mathrm{R} \$ 724,00$ ), por meio da pergunta: "Qual a renda mensal de sua família?". As opções de respostas foram: até 2 salários mínimos, de 2 a 5 salários, de 5 a 10 salários, > que 10 salários e não sei. Optou-se por agrupar a renda em três categorias (até 2 salários, de 2 a 5 salários e $>5$ salários), pois apenas $41(4,0 \%)$ estudantes responderam que a renda familiar mensal era superior a 10 salários.

O nível de atividade física foi estimado por meio de uma questão extraída do questionário Youth Risk Behavior Surveillance System(YRBSS ${ }^{20}$ devidamente traduzido e validado para o Brasil ${ }^{21}$. A questão consiste na seguinte pergunta: "Durante os últimos sete dias, em quantos dias você foi ativo fisicamente por pelo menos 60 minutos por dia?" (Considere o tempo que você gastou em qualquer tipo de atividade física que aumentou sua frequência cardíaca e fez com que sua respiração ficasse mais rápida por algum tempo). As sugestões de respostas variaram entre zero (nenhum dia) e sete dias. Os pontos de corte da Organização Mundial de Saúde ${ }^{22}$ foram adotados para classificar o nível de atividade física dos adolescentes, sendo 60 minutos diários de AF para os adolescentes de 15 a 17 anos e 150 minutos semanais para os adolescentes com idade igual ou superior a 18 anos. A atividade física foi categorizada em atende ou não atende às recomendaçôes.

O IMC (massa corporal/estatura ${ }^{2}$ ) foi calculado a partir da aferição da massa corporal e estatura dos adolescentes. A massa corporal foi mensurada seguindo a padronização da International Society for the Advancement of Kinanthropometry(ISAK) ${ }^{23}$, por meio de uma balança digital da marca G Tech Pro, com capacidade de até $150 \mathrm{~kg}$ e resolução de 100 gramas. Para a aferição da estatura, utilizou-se um estadiômetro da marca Sanny com resolução de 0,1 $\mathrm{cm}$. O IMC foi classificado em quatro categorias (baixo peso, peso normal, sobrepeso e obesidade) de acordo com os pontos de cortes específicos para adolescentes que levaram em consideração o sexo e a idade ${ }^{24}$. Neste estudo, a proporção de adolescentes com baixo peso foi de $6,9 \%$, assim optou-se por agrupá-los em peso normal juntos com os adolescentes eutróficos $(62,4 \%)$. Do mesmo modo, os adolescentes com sobrepeso $(14,4 \%)$ e os obesos 
(16,3\%) foram agrupados em excesso de peso.

Todas as análises foram conduzidas no software The Statistical Package for the Social Sciences (SPSS), versão 20.0, considerando nível de significância de 5\%. Foram empregadas análises descritivas (frequências absoluta e relativa) e inferenciais. $\mathrm{O}$ teste qui-quadrado foi utilizado com intuito de identificar possíveis associações entre as variáveis. $\mathrm{Na}$ análise de associação, bruta e ajustada, foi empregada a regressão logística binária para estimar a razão de odds (RO) e os intervalos de confiança de 95\% (IC95\%). Na análise ajustada, foram incluídas todas as variáveis simultaneamente no modelo.

\section{Resultados}

Foram coletadas informaçóes de 1.148 adolescentes de ambos os sexos, regularmente matriculados nas escolas públicas selecionadas para o presente estudo. Do total de alunos que estavam em aula nos dias da coleta e tiveram suas informaçóes coletadas, 119 não foram considerados elegíveis no presente estudo (66 por apresentarem idade inferior a 15 anos, 16 por terem idade igual ou superior a 20 anos, 24 por não terem respondido a questão referente ao deslocamento para a escola e 13 por terem respondido outros na variável dependente).
Assim, a amostra final foi composta por 1.029 adolescentes de 15 a 19 anos (média=16,3 anos; $\mathrm{dp}=1,04)$, dos quais $54,7 \%$ eram do sexo feminino.

A maioria dos estudantes tinha idade entre 15 e 16 anos (57,4\%), estudava no período diurno $(72,2 \%)$, na primeira série do ensino médio $(38,9 \%)$ e era de família com renda mensal de dois a cinco salários (39,6\%). Observou-se, de modo geral, uma grande proporção de adolescentes com excesso de peso $(30,7 \%)$ e que não atendiam às recomendaçóes de atividade física $(88,2 \%)$ (TABELA 1$)$.

TABELA 1 -Características gerais dos adolescentes $(\mathrm{n}=1.029)$ na amostra total e estratificado por sexo. São José, Santa Catarina, Brasil, 2014.

\begin{tabular}{|c|c|c|c|}
\hline \multirow{3}{*}{ Variáveis } & \multirow{2}{*}{ Amostra total } & \multicolumn{2}{|c|}{ Sexo } \\
\hline & & Masculino & Feminino \\
\hline & n (\%) & n $(\%)$ & n (\%) \\
\hline \multicolumn{4}{|l|}{ Idade (anos) } \\
\hline $15-16$ & $590(57,3)$ & $255(43,2)$ & $335(56,8)$ \\
\hline $17-19$ & $439(42,7)$ & $211(48,1)$ & $228(51,9)$ \\
\hline \multicolumn{4}{|l|}{ Turno } \\
\hline Diurno & $744(72,7)$ & $328(44,1)$ & $416(55,9)$ \\
\hline Noturno & $280(27,3)$ & $135(48,2)$ & $145(51,8)$ \\
\hline \multicolumn{4}{|l|}{ Série } \\
\hline Primeiro ano & $398(38,9)$ & $171(43,0)$ & $227(57,0)$ \\
\hline Segundo ano & $331(32,4)$ & $137(46,6)$ & $157(53,4)$ \\
\hline Terceiro ano & $294(28,7)$ & $154(46,5)$ & $177(53,5)$ \\
\hline \multicolumn{4}{|l|}{ Renda Familiar } \\
\hline$<$ de 2 salários & $237(27,8)$ & $79(33,3)$ & $158(66,7)$ \\
\hline Entre 2 e 5 salários & $406(47,5)$ & $187(46,1)$ & $219(53,9)$ \\
\hline$\geq$ de 5 salários & $211(24,7)$ & $118(55,9)$ & $93(44,1)$ \\
\hline \multicolumn{4}{|l|}{ IMC } \\
\hline Peso normal & $713(69,3)$ & $341(47,8)$ & $372(55,2)$ \\
\hline Excesso de peso & $316(30,7)$ & $125(39,6)$ & $191(60,4)$ \\
\hline \multicolumn{4}{|l|}{ Atividade Física } \\
\hline Não atende às recomendações & $823(88,2)$ & $386(46,9)$ & $437(53,1)$ \\
\hline Atende as recomendaçóes & $110(11,8)$ & $28(25,5)$ & $82(74,5)$ \\
\hline
\end{tabular}

$\mathrm{n}$ : frequência absoluta;

(\%): frequência relativa; IMC: Índice de Massa Corporal; min: minutos. 
Verificou-se que 52,0\% (IC95\%= 48,8-54,6) dos adolescentes se deslocavam de maneira passiva para a escola, sendo superior nas moças $(56,5 \%)$ em relação aos rapazes (46,6\%). Maior prevalência do deslocamento passivo foi observada nos adolescentes mais jovens, de 15 e 16 anos $(51,1 \%)$ e de famílias com maior renda familiar $(63,5 \%)$. Destaca-se que os adolescentes que se deslocavam passivamente para a escola, apresentaram maior prevalência de excesso de peso $(56,6 \%)$ e não atende às recomendaçôes de atividade físicas $(53,9 \%)$. Os adolescentes que despendiam maior tempo no deslocamento para a escola (> de 20 minutos) se deslocavam de forma passiva $(64,1 \%)$ (TABELA 2).

TABELA 2-Características gerais dos adolescentes de acordo com o deslocamento para a escola $(n=1029)$. São José, Santa Catarina, Brasil, 2014.

\begin{tabular}{|c|c|c|c|c|}
\hline Variáveis & $\begin{array}{r}\text { Total } \\
\text { n }(\%) \\
\end{array}$ & $\begin{array}{c}\text { Ativo }(\mathrm{n}=494) \\
\mathrm{n}(\%)\end{array}$ & $\begin{array}{c}\text { Passivo }(\mathrm{n}=535) \\
\text { n }(\%)\end{array}$ & p-valor \\
\hline Sexo & & & & 0,002 \\
\hline Masculino & $466(45,3)$ & $249(53,4)$ & $217(46,6)$ & \\
\hline Feminino & $563(54,7)$ & $245(43,5)$ & $318(56,5)$ & \\
\hline Idade (anos) & & & & 0,875 \\
\hline $15-16$ & $590(57,3)$ & $282(47,8)$ & $308(52,2)$ & \\
\hline $17-19$ & $439(42,7)$ & $212(48,3)$ & $227(51,1)$ & \\
\hline Renda Familiar (salários) & & & & 0,001 \\
\hline$>$ de 5 & $211(24,7)$ & $77(36,5)$ & $143(63,5)$ & \\
\hline De 2 a 5 & $406(47,5)$ & $199(49,0)$ & $207(51,0)$ & \\
\hline$<\operatorname{de} 2$ & $237(27,8)$ & $129(54,4)$ & $108(45,6)$ & \\
\hline IMC & & & & 0,047 \\
\hline Peso normal & $713(69,3)$ & $357(50,1)$ & $356(49,9)$ & \\
\hline Excesso de peso & $316(30,7)$ & $137(43,4)$ & $179(56,6)$ & \\
\hline Atividade Física & & & & 0,001 \\
\hline Não atende às recomendaçôes & $823(88,2)$ & $379(46,1)$ & $444(53,9)$ & \\
\hline Atende às recomendaçóes & $110(11,8)$ & $70(63,6)$ & $40(36,4)$ & \\
\hline Tempo de Deslocamento (min) & & & & $<0,001$ \\
\hline$<10$ & $311(30,4)$ & $123(35,9)$ & $220(64,1)$ & \\
\hline Entre 10 e 19 & $369(36,1)$ & $191(51,8)$ & $178(48,2)$ & \\
\hline$\geq \operatorname{de} 20$ & $343(33,5)$ & $177(56,9)$ & $134(43,1)$ & \\
\hline
\end{tabular}

As análises de regressão foram realizadas de acordo com o sexo separadamente conforme as TABELAS 3 e 4, (masculino e feminino, respectivamente). Após os ajustes, observou-se no sexo masculino, que os adolescentes com mais idade (17-19 anos) tiveram maior razão de Odss ( $\mathrm{OR}=1,69$; IC95\%= 1,06-2,69) de se deslocar passivamente para a escola quando comparados aos mais novos (15-16 anos). Em relação à renda familiar, tantos os rapazes quanto às moças que apresentaram renda superior a cinco salários, apresentaram maiores razóes de $\mathrm{Odss}(\mathrm{OR}=2,74$; IC95\% $=1,42-5,30 ;$ OR $=1,98 ;$ IC95\%= 1, 10-3,55, respectivamente) de se deslocar de maneira passiva para a escola em relação aos seus respectivos pares com renda mensal de até dois salários mínimos.

O nível de atividade física também se associou ao deslocamento passivo, pois em ambos os sexos, os adolescentes que não atenderam às recomendações de atividades físicas apresentaram maiores razões de Odss (OR=3,72; IC95\%=1,39-9,93, para os rapazes e $\mathrm{OR}=2,21 ; \mathrm{IC} 95 \%=1,26-3,87$, para as moças) de se deslocarem de maneira passiva para a escola em relação àqueles que atendiam às recomendações. O tempo de deslocamento para a escola esteve associado ao deslocamento passivo em ambos os sexos. Observou-se que os rapazes com maior tempo (> de 20 minutos) gasto no deslocamento para a escola, apresentaram maior razão de Odss (OR= 2,55 IC95\%= 1,55-4,48) de se deslocar passivamente em relação aqueles com menor atempo (< de 10 minutos). Entre as moças, aquelas que se dispendiam maior tempo no
* Teste de quiquadrado. 
Pinto AA, et al.

IMC: Índice de Massa Corporal; mim: minutos.

* Ajustada por todas as variáveis.

IMC: Índice de Massa Corporal; mim: minutos.

* Ajustada por todas as variáveis. deslocamento (entre 10 e 19 minutos e mais de 20 minutos), apresentaram, respectivamente, maiores razōes de Odss OR=1,70; IC95\%= 1,06-2,75 e
$\mathrm{OR}=2,99 ; \mathrm{IC} 95 \%=1,78-5,02$, de se deslocarem de maneira passiva para a escola quando comparados aquelas com menor tempo (menos de 10 minutos).

TABELA 3 -Análise de regressão logística para identificação de fatores associados ao deslocamento passivo para ir à escola de adolescentes do sexo masculino. São José, Santa Catarina, Brasil, 2014.

\begin{tabular}{|c|c|c|c|}
\hline \multirow{2}{*}{ Variáveis } & Deslocamento Passivo & Análise bruta & Análise ajustada* \\
\hline & $(\%)$ & RO (IC95\%) & RO (IC95\%) \\
\hline \multicolumn{4}{|l|}{ Idade (anos) } \\
\hline $17-19$ & 48,3 & $0,88(0,61-1,27)$ & $1,69(1,06-2,69)$ \\
\hline $15-16$ & 45,1 & 1 & 1 \\
\hline \multicolumn{4}{|l|}{ Renda Familiar } \\
\hline$>$ de 5 salários & 59,3 & $2,41(1,40-4,16)$ & $2,74(1,42-5,30)$ \\
\hline Entre 2 e 5 salários & 42,8 & $1,44(0,83-2,49)$ & $1,48(0,82-2,69)$ \\
\hline$<$ de 2 salários & 34,2 & 1 & 1 \\
\hline \multicolumn{4}{|l|}{ IMC } \\
\hline Excesso de peso & 48,0 & $1,08(0,72-1,63)$ & $0,77(0,46-1,29)$ \\
\hline Peso normal & 46,0 & 1 & 1 \\
\hline \multicolumn{4}{|l|}{ Atividade Física } \\
\hline Não atende às recomendaçôes & 48,4 & $3,85(2,10-7,07)$ & $3,72(1,39-9,93)$ \\
\hline Atende às recomendaçóes & 25,0 & 1 & 1 \\
\hline \multicolumn{4}{|l|}{ Tempo de Deslocamento (min) } \\
\hline$\geq \operatorname{de} 20$ & 62,4 & $2,98(1,87-4,75)$ & $2,55(1,45-4,48)$ \\
\hline $10-19$ & 40,8 & $1,23(0,78-1,96)$ & $1,52(0,87-2,67)$ \\
\hline$<$ de 10 & 35,8 & 1 & 1 \\
\hline
\end{tabular}

TABELA 4 -Análise de regressão logística para identificação de fatores associados ao deslocamento passivo para ir à escola de adolescentes do sexo feminino. Florianópolis, Santa Catarina, Brasil, 2014.

\begin{tabular}{|c|c|c|c|}
\hline \multirow{2}{*}{ Variáveis } & Deslocamento Passivo & Análise bruta & Análise ajustada* \\
\hline & $(\%)$ & RO (IC95\%) & RO (IC95\%) \\
\hline \multicolumn{4}{|l|}{ Idade (anos) } \\
\hline $15-16$ & 57,6 & $1,12(0,80-1,57)$ & $1,08(0,71-1,64)$ \\
\hline $17-19$ & 54,8 & 1 & 1 \\
\hline \multicolumn{4}{|l|}{ Renda Familiar } \\
\hline$>$ de 5 salários & 68,8 & $1,39(0,91-2,14)$ & $1,98(1,10-3,55)$ \\
\hline Entre 2 e 5 salários & 58,0 & $1,31(0,87-1,98)$ & $1,31(0,84-2,05)$ \\
\hline$<$ de 2 salários & 51,3 & 1 & 1 \\
\hline \multicolumn{4}{|l|}{ IMC } \\
\hline Excesso de peso & 62,3 & $1,44(1,01-2,05)$ & $0,71(0,47-1,19)$ \\
\hline Peso normal & 53,5 & 1 & 1 \\
\hline \multicolumn{4}{|l|}{ Atividade Física } \\
\hline Não atende às recomendaçóes & 58,8 & $1,72(0,90-3,28)$ & $2,21(1,26-3,87)$ \\
\hline Atende às recomendaçôes & 40,2 & 1 & 1 \\
\hline \multicolumn{4}{|l|}{ Tempo de Deslocamento (min) } \\
\hline$\geq \operatorname{de} 20$ & 65,6 & $1,93(1,25-2,97)$ & $2,99(1,78-5,02)$ \\
\hline $10-19$ & 53,8 & $1,18(0,78-1,77)$ & $1,70(1,06-2,75)$ \\
\hline$<\operatorname{de} 10$ & 49,7 & 1 & 1 \\
\hline
\end{tabular}




\section{Discussão}

Considerando o deslocamento passivo para a escola como desfecho, o presente estudo evidenciou que pouco mais da metade dos adolescentes deslocavam-se passivamente para ir à escola. Os fatores associados com o deslocamento passivo foram a faixa etária nos rapazes, e as variáveis renda familiar, atividade física e o tempo gasto no trajeto casa-escola em ambos os sexos.

Pesquisas prévias que investigaram o deslocamento para a escola na adolescência, encontraram prevalências superiores de deslocamento passivo. $\mathrm{Na}$ Alemanha, Reimers, et al. ${ }^{10}$ encontrou prevalência superior de deslocamento passivo para a escola $(58,3 \%)$ quando comparado aos achados do presente estudo (52,0\%). Em adolescentes americanos, constatou-se que quase todos os adolescentes $(95,0 \%)$ se deslocavam de forma passiva ${ }^{9}$. Em adolescentes portugueses também foi observado uma prevalência superior $(78,6 \%)^{12}$. No Brasil, foram observadas prevalências inferiores aos achados do presente estudo quanto ao deslocamento passivo em Santa Catarina $(43,3 \%)^{6}$, Pernambuco $(43,0 \%)^{13}$ e Rio Grande do Sul $(35,3 \%)^{11}$.

Para as divergências encontradas nas prevalências de deslocamento passivo entre os estudos, é necessário considerar as faixas etárias utilizadas, pois padrões de comportamento na adolescência podem variar conforme a idade ${ }^{14}$. Deste modo, quanto aos estudos que envolveram adolescentes com uma faixa etária muito ampla, é preciso atentar para o fato de que quanto mais jovem o adolescente, menor é o poder de decisão dele em relação àqueles adolescentes que se encontram no final desse período etário ${ }^{4}$. Além disso, é preciso considerar questóes que podem ser primordiais para a tomada de decisão quanto à forma de adoção dos modos de deslocamento, com destaque para a falta de segurança e de acessibilidade, e ausência de políticas públicas que incentivem o deslocamento ativo ${ }^{25}$. SANTOS, et al. ${ }^{13}$, ao investigar adolescentes pernambucanos (14 a 19 anos), chamam a atenção para outras variáveis que podem explicar a adesão da forma de deslocamento para a escola, como posse de carteira de habilitação e do número de veículos motorizados presentes na residência.

A faixa etária (17-19 anos) se associou ao deslocamento passivo entre os rapazes, sugerindo que aqueles mais velhos têm mais chances de se deslocar passivamente para a escola do que os mais novos. Estes resultados corroboram os achados apresentados no estudo de CHILlon, et al. ${ }^{26}$, conduzido em 2.183 adolescentes espanhóis. Resultados semelhantes foram encontrados em estudo realizado com 628 adolescentes da Alemanha corrobora os resultados encontrados na presente investigação ${ }^{27}$. Resultado divergente, no entanto, foi observado em estudo com 4.207 adolescentes pernambucanos ${ }^{13}$. Em alguns países da Europa e da América é comum a posse da carteira de habilitação ainda muito jovem (14 e 16 anos $)^{28}$, sendo provável que os adolescentes com idade mais avançada sejam menos ativo no deslocamento para a escola do que os mais jovens. Entre os adolescentes do presente estudo, é presumível que esta associação esteja atrelada às atividades extracurriculares, pois geralmente os adolescentes do ensino médio, principalmente aqueles em fase de conclusão, estão envolvidos em estágios remunerados no contraturno escolar e também em cursos pré-vestibulares, assim, para otimizar o tempo entre as funçōes extracurriculares e acadêmicas, os adolescentes mais velhos optem pelo deslocamento passivo.

Os resultados do presente estudo mostraram que os adolescentes de famílias com maior renda mensal apresentaram maiores chances de se deslocar passivamente para a escola. Essa associação foi evidenciada em adolescentes (13 a 17 anos) da Alemanha, sendo que àqueles de famílias com melhores condiçóes financeiras eram mais propensos a se deslocarem de forma passiva para a escola ${ }^{10}$. Outro estudo, realizado com adolescentes (15 a 19 anos) de Santa Catarina, verificou que os de família com menor renda, eram mais propensos a se deslocarem de forma ativa para a escola do que seus pares provenientes de famílias mais abastadas ${ }^{6}$.

Coincidentemente, no presente estudo os rapazes que se deslocavam de forma passiva para a escola eram, em sua maioria $(55,6 \%)$, de famílias com maior renda mensal (> 5 salários) e àqueles que apresentavam maior número de automóveis (dados não apresentados). Desse modo, o número de automóveis na residência pode explicar essa associação, uma vez que este tem sido sugerido como um indicador de melhor condição financeira ${ }^{10}$. Além disso, os estudantes de escolas públicas brasileiras são geralmente provenientes de famílias de média/baixa renda, sendo provável que o deslocamento ativo seja mais frequente entre esses estudantes ${ }^{6}$. Assim, acredita-se que os resultados 
encontrados no presente estudo retrataram a realidade em relação às questões financeiras, evidenciando que os adolescentes com maior renda mensal são mais propensos a se deslocarem de maneira passiva para a escola, mesmo sendo de escolas públicas.

Os resultados encontrados no presente estudo evidenciaram, ainda, que o deslocamento passivo para a escola esteve associado à atividade física, os quais apontam que os adolescentes que não atendem às recomendações de AF apresentaram maiores chances de se deslocar passivamente à escola. No entanto, é preciso atentar para o fato de que no presente estudo a proporção de adolescentes que atendiam às recomendações foi bastante elevada $(88,2 \%)$, o que provavelmente pode ter contribuído para esse resultado. Apesar disso, inúmeros estudos que investigaram o deslocamento para a escola reforçam a importância desse domínio da $\mathrm{AF}$ no atendimento às recomendaçōes de AF necessária para ganhos positivos e/ou manutenção da saúde ${ }^{4,5,7,8}$. Deste modo, entendese que, principalmente nos dias atuais, deslocarse de maneira ativa é importante, visto que uma redução dos níveis de atividades físicas entre escolares tendem a reduzir, principalmente na adolescência ${ }^{2}$. É importante salientar, que apesar de haver outros fatores responsáveis pela adesão do deslocamento passivo, é provável que os adolescentes desconheçam que o deslocamento é um domínio da AF capaz de contribuir para atendimento a tais recomendações.

Em relação ao tempo dispendido no trajeto de casa até a escola, observou-se que quanto maior o tempo gasto para se deslocar até a escola maior a chance dos adolescentes se deslocarem de forma passiva. Um estudo realizado com adolescentes (11 a 17 anos) da cidade de Caxias do Sul-RS constatou que a maior frequência do deslocamento passivo para a escola ocorria entre as viagens com tempo igual ou superior a 20 minutos $^{11}$.

Uma possível explicação para tais achados parece estar relacionada à distância entre a residência e a escola. Harten e OLds ${ }^{29}$ em estudo com adolescentes australianos, ao considerarem a distância entre a residência e a escola, verificaram maior ocorrência do deslocamento ativo $(90,0 \%)$ quando a distância neste percurso era igual ou inferior a 250 metros. Corroborando estes dados, um estudo conduzido com adolescentes irlandeses, constatou redução do deslocamento ativo em $71 \%$ ao passo em que uma milha era acrescentada a distância entre a residência e a escola ${ }^{14}$. Os resultados encontrados no presente estudo estão de acordo com a hipótese levantada por SiLVA, Lopes e SiLVA ${ }^{19}$, que parece ser provável que as cidades cresçam mais rapidamente do que o número de novas escolas, o que pode estar associado ao aumento da distância entre as novas residências e as escolas e, consequentemente, ao tempo gasto no deslocamento passivo neste percurso.

As principais limitações deste estudo incluem a ausência de informações de estudantes da rede privada, retratando um único cenário de ensino. $\mathrm{Na}$ pergunta para o deslocamento ativo, não foi permitido o registro do tipo de deslocamento utilizado entre aqueles que responderam outros deslocamentos. No entanto, poucos adolescentes relataram essa categoria. O tempo despendido foi registrado em faixas de tempo, o que de certo modo limita uma análise mais detalhada desse aspecto. Não foi possível coletar a distância entre a residência e a escola, o que poderia ajudar a entender a relação encontrada com a variável tempo. A renda familiar foi coletada em faixas, o que aumenta a possibilidade de heterogeneidade dos grupos, quanto a sua realidade econômica.

Dentre os pontos fortes, destaca-se o uso de técnica de amostragem probabilística e representativa de adolescentes do município investigado. Por fim, os achados desta investigação podem servir para reforçar as evidências encontradas na literatura, no que diz respeito ao nível de AF e o tipo de deslocamento comumente utilizado pelos adolescentes para ir à escola.

De acordo com os resultados, a cada dez adolescentes, cinco deslocam-se passivamente para ir à escola. Quanto maior tempo gasto no deslocamento, o não atendimento às recomendações em ambos os sexos, e às condições financeiras nos rapazes, maior a possibilidade dos adolescentes se deslocarem de modo passivo.

Sugere-se a comunidade escolar (diretores, professores de sala de aula e de Educação Física), que empoderem aos alunos e suas famílias sobre os benefícios que o deslocamento ativo para ir à escola pode trazer a sua saúde e bem-estar, incentivandoos a adotarem esse comportamento. Além disso, sugere-se o desenvolvimento de políticas públicas voltadas para o incentivo do deslocamento ativo para a escola, bem como investimentos do setor público para construção de ciclo-faixas, melhora das condiçôes das calçadas, de segurança nos bairros e ampliação do número de novas escolas entre aqueles bairros mais distantes. 


\title{
Conflito de interesse
}

Nada à declarar.

\begin{abstract}
Physical inactivity in commuting to school and associated factors in adolescents in a city in Southern Brazil

The objective of this study was to estimate the prevalence of physical inactivity in commuting to school and identify associated factors in adolescents in a city in Southern Brazil. Epidemiological study conducted in 1.029 adolescents aged 15 to 19 years, from public state schools of the city of São José-SC. A questionnaire was applied in order to obtain information regarding the dependent variable, passive commuting to school (car / motorcycle, bus) and the independent variables, and sociodemographic characteristics (gender, age and family income), physical activity and time spent in commuting. Body weight and height were measured to determine the status of the weight using the body mass index. He used binary logistic regression, estimating the ratio Odss (RO) to identify the association between the dependent variable and the other variables. The prevalence of passive commuting among adolescents was 52\%. Older boys (17-19 years), the adolescents from families with greater monthly family income ( $>5$ salaries), to those who did not meet the recommendations of physical activity and those who spent more time on commuting to school ( $\geq 20$ minutes for the boys and $\geq 10$ minutes for girls) presented more chances to move passively. More than half of the adolescents moving from passive manner at home-school trajectory. The groups that are most exposed to passive commuting are older boys, adolescents with higher family incomes, which does not meet the recommendations in the world of physical activity and spend more time on displacement.
\end{abstract}

KEYWORDS: Motor Activity; Walking; Bicycling; Adolescent Health.

\section{Referências}

1. Lee I-M, Shiroma EJ, Lobelo F, Puska P, Blair SN, Katzmarzyk PT. Effect of physical inactivity on major noncommunicable diseases worldwide: an analysis of burden of disease and life expectancy. Lancet. 2012;380(9838):219229. doi: 10.1016/S0140-6736(12)61031-9.

2. Hallal PC, Andersen LB, Bull FC, Guthold R, Haskell W, Ekelund U. Global physical activity levels: surveillance progress, pitfalls, and prospects. Lancet. 2012;380(9838):230- 240. doi: 10.1016/ S0140-6736(12)60633-3.

3. World Health Organization. Global strategy on diet, physical activity and health. Geneva: WHO; 2004.

4. Baig F, Hameed MA, Li M, Shorthouse G, Roalfe AK, Daley A. Association between active commuting to school, weight and physical activity status in ethnically diverse adolescents predominately living in deprived communities. Public Health. 2009;123:39- 41. doi: 10.1016/j.puhe.2008.08.004.

5. Larouche R, Saunders TJ, Faulkner GEJ, Colley RC, Tremblay MS. Associations between active school transport and physical activity, body composition and cardiovascular itness: a systematic review of 68 studies. J Phys Act Health. 2014;11:206-211. doi: 10.1123/ jpah.2011-034.

6. Silva KS, Nahas MV, Borgato AF, Oliveira ES, Del Duca GF, Lopes AS. Factors associated with active commuting to school and to work among Brazilian adolescents. J Phys Act Health. 2011;8(7):926- 933.

7. $\mathrm{Xu} \mathrm{H}$, Wen LM, Rissel C. The relationships between active transport to work or school and cardiovascular health or body weight: asystematic review. Asia Pac J Public Health. 2013;25(4):298-315. doi: 10.1177/1010539513482965.

8. Saunders L, Green J, Petticrew M, Steinbach R, Roberts H. What are the health benefits of active travel? A systematic review of trials and cohort studies. PLoS One. 2013;8(8):e69912. doi: 10.1371/journal.pone.0069912.

9. Bungum TJ, Lounsbery M, Moonie S, Gast J. Prevalence and correlates of walking and biking to school among adolescents. J Community Health. 2009;34:129- I34. doi: 10.1007/s10900-008-9135-3. 
10. Reimers AK, Jekauc D, Peterhans E, Wagner MO, Woll A. Prevalence and socio-demographic correlates of active commuting to school in a nationwide representative sample of German adolescents. Prev Med. 2013;56(1):64- 69. doi: 10.1016/j.ypmed.2012.11.011.

11. Silva KS, Vasques DG, Martins CO, Williams LA, Lopes AS. Active commuting: prevalence, barriers, and associated variables. J Phys Act Health. 2011;8:750-757.

12. Aires L, Pratt M, Lobelo F, Santos RM, Santos MP, Mota J. Associations of cardiorespiratory fitness in children and adolescents with physical activity, active commuting to school, and screen time. J Phys Aet Health. 2011;8(Suppl. 2):S198-S205.

13. Santos KM, Júnior RSW, Barros SSH, Júnior JCR, Barros MGB. Prevalence of physical inactivity and associated factors among adolescents commuting to school. Cad Saúde Pública. 2010;26(7):1419-1430.

14. Nelson NM, Foley E, O'Gorman DJ, Moyna NM, Woods CB. Active commuting to school: how far is too far? Int J Behav Nutr Phys Act. 2008;8:51. doi: 10.1186/1479-5868-5-1.

15. Programa das Naçôes Unidas para o Desenvolvimento. IDH-M. Índice de Desenvolvimento Humano-Municipal [Internet]. Brasília: PNUD; 2010 [citado 8 out 2015]. Disponível em: http://www.pnud.org.br/atlas/ ranking/RankingIDHM-Municipios-2010.aspx.

16. Luiz RR, Magnanini MMF. A lógica da determinação do tamanho da amostra em investigaçóes epidemiológicas. Cad Saúde Colet. 2000;8(2):9-28.

17. Kuhnen M, Boing AF, Oliveira MC de, Longo GZ, Njaine K. Smoking and associated factors in Brazilian adults: a population-based study. BMC Oral Health. 2009;25:9-7. doi: 10.1186/1472-6831-9-7.

18. World health organization. Organización Mundial de La Salud. La salud de los jóvenes: un reto y una esperanza. Genebra: WHO; 1995.

19. Silva KS, Lopes AS, Silva FM. Atividade física no deslocamento à escola e no tempo livre em crianças e adolescentes da cidade de João Pessoa, PB, Brasil. Rev Bras Ciênc Mov. 2007;15:61-70.

20. Eaton DK, Kann L, Kinchen S, Shanklin S, Ross J, Hawkins J, et al. Youth risk behavior surveillance-United States, 2007. MMWR Surveill Summ. 2008;57(4):1-131.

21. Guedes DP, Lopes CC. Validação da versão brasileira do youth risk behavior survey 2007. Rev Saude Publica. 2010;44:840-850.

22. World Health Organization. Global Recommendations on Physical Activity for Health. Geneva: WHO; 2010.

23. Marfell-Jones M, Olds T, Stewart A, Carter L. International standards for anthropometric assessment. Potchefstroom, South Africa: ISAK; 2006.

24. Cole TJ, Lobstein T. Extended international (IOTF) body mass index cut-offs for thinness, overweight and obesity. Pediatr obes. 2012;7(4):284-294.

25. Benedet, J, Assis MAA, Calvo MCM, Andrade DF. Excesso de peso em adolescentes: explorando potenciais fatores de risco. Rev Paul Pediatr. 2013;31(2):172-81.

26. Chillón P, Ortega FB, Ruiz JR, Pérez IJ, Martín-Martillas M, Valtueña J, et al. Socio-economic factors and active commuting to school in urban Spanish adolescents: the AVENA study. Eur J Public Health. 2009;19:470-476. doi:10.1093/eurpub/ckp048.

27. Landsberg B, Plachta-Danielzik S, Much D, Johannsen N, Lange D, Müller MJ. Associations between active commuting to school, fat mass and lifestyle factors in adolescents: the Kiel Obesity Prevention Study. EurJ Clin Nutr. 2007;62:739747. doi: 10.1038/sj.ejcn.1602781.

28. Davison KK, Werder JL, Lawson CT. Children's active commuting to school: Current knowledge and future directions. Prev Chronic Dis. 2008;5(3):A100.

29. Harten N, Olds T. Physical activity: patterns of active transport in 11-12 year old Australian children. Aust N Z J Public Health. 2004;28(2):167-72.

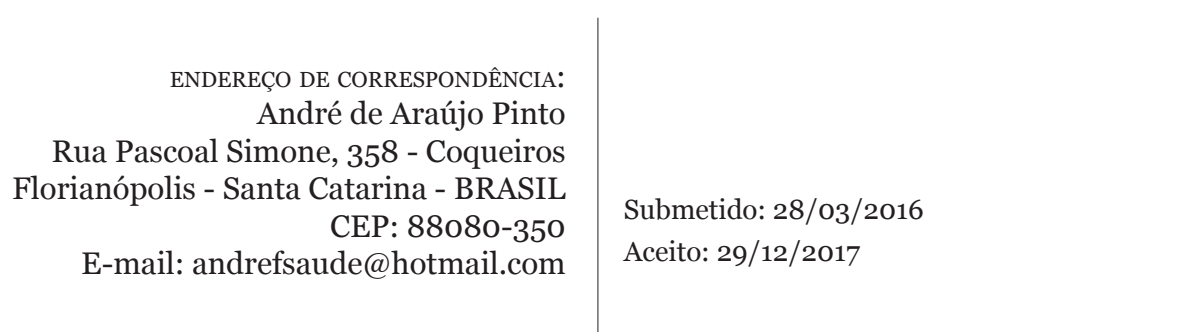

